\title{
Hyponatremia in the Emergency Department: Could Biomarkers Help in Diagnosis and Treatment?
}

\author{
Karin Olsson ${ }^{*}$, Sofia Enhörning², Bertil Öhlin², Olle Melander ${ }^{2}$ \\ ${ }^{1}$ Department of Endocrinology, Skane University Hospital, Lund, Sweden \\ ${ }^{2}$ Department of Clinical Science, Lund University, Lund, Sweden \\ Email: *Karin.C.Olsson@skane.se
}

Received 18 January 2016; accepted 19 March 2016; published 22 March 2016

Copyright (C) 2016 by authors and Scientific Research Publishing Inc.

This work is licensed under the Creative Commons Attribution International License (CC BY). http://creativecommons.org/licenses/by/4.0/

(c) (i) Open Access

\begin{abstract}
Objective: Hyponatremia is the most common electrolyte imbalance. The initial treatment decision is based on clinical evaluation of patient volume status but an accurate assessment is difficult, particularly differentiating mild hypovolemia from euvolemia. The aim of this study is to examine if biomarkers are valuable in the early determination of volume status and SIADH diagnosis. Methods: Blood samples were collected from an unselected patient population at entry to the Emergency Department. If the plasma sodium level (P-Na) was $\leq 125 \mathrm{mmol} / \mathrm{L}$, the sample was frozen for further analysis. Mid-regional pro-atrial natriuretic peptide (MR-proANP), proadrenomedullin (MR-proADM), C-terminal prepro-vasopressin (copeptin), pro-endothelin-1 (proET-1) and N-terminal pro-brain natriuretic peptide (NT-proBNP) levels were analysed. A comprehensive assessment of volume status and underlying causes was made after discharge blinded for biomarker results. Results: A total of 81 patients were included. A well substa ntiated volemic state (hypo/eu/hypervolemia) was established in 72 patients (mean age 76 years, 65\% women, median P-Na $119 \mathrm{mmol} / \mathrm{L}$ ). A significant association was observed between MR-proANP levels and volemic state $(p=0.0001)$. Data was specifically analysed with respect to distinguishing hypo- from euvolemia $(n=59)$ using logistic regression. In a crude analysis, MR-proANP was significantly related to euvolemia (OR: 2.54 per SD of MR-proANP, 95\% CI $1.32-4.86, p=0.005$ ) and remained so after the multivariate backward elimination model (OR: 2.45 per SD of MRproANP, 95\% CI 1.22 - 4.91, $p=0.012$.), whereas the other studied biomarkers were not. Copeptin levels were not associated with a diagnosis of SIADH. Conclusions: MR-proANP may be of value in early determination of volume status in hyponatremic patients.
\end{abstract}

\section{Keywords}

Hyponatremia, SIADH, ANP, Copeptin

"Corresponding author.

How to cite this paper: Olsson, K., Enhörning, S., Öhlin, B. and Melander, O. (2016) Hyponatremia in the Emergency Department: Could Biomarkers Help in Diagnosis and Treatment? Open Journal of Emergency Medicine, 4, 11-22. 


\section{Introduction}

Hyponatremia, being the most common electrolyte disturbance in the Emergency Department with a prevalence of approximately $3 \%$, has a complex pathogenesis [1] [2]. Thus, multiple regulatory systems are involved in the meticulous maintenance of osmolarity as well as volume status.

In an ideal clinical setting management, chronic hyponatremia is based on an robust and accurate assessment of volume status, a challenging task for the clinician, especially when differentiating euvolemia from mild hypovolemia (Figure 1). Previous studies have demonstrated that clinical assessment frequently is inaccurate, and no gold standard exists for the categorization of hyponatremic patients into volume status groups (hypo-, eu- and hypervolemia) [3] [4].

This major impediment in clinical management may delay an early and proper diagnosis and thereby hinders expedient and effective treatment, especially in euvolemic patients with SIADH (syndrome of inappropriate ADH secretion), where the development of vasopressin-receptor antagonists lately has been added to the armamentarium as an physiological treatment option.

The aim of the present study is to evaluate if biomarkers can aid early determination of volume status and the diagnosis of SIADH in hyponatremic patients.

The regulation of sodium balance is complex and the result of several regulatory systems acting both in unison and in discord. Osmoreceptors in the hypothalamus reacts to changes in osmolality and baroreceptors in the aorta responds to changes in blood volume communicating with the hypothalamus thereby influencing the secretion of vasopressin. Natriuretic peptides produced in the heart affect sodium reabsorption in the kidney.

We hypothesize that circulating hormonal biomarkers sensitive to cardiovascular pressure load (NT-proBNP, MR-proANP), vascular stress (pro-ET, MR-proADM), and regulation of sodium balance and osmolality (copeptinas a surrogate for vasopressin excretion) can be useful as diagnostic tools. Levels of mid-regional pro-atrial natriuretic peptide (MR-proANP), copeptin, mid-regional pro-adrenomedullin (MR-proADM), pro-endothelin-1

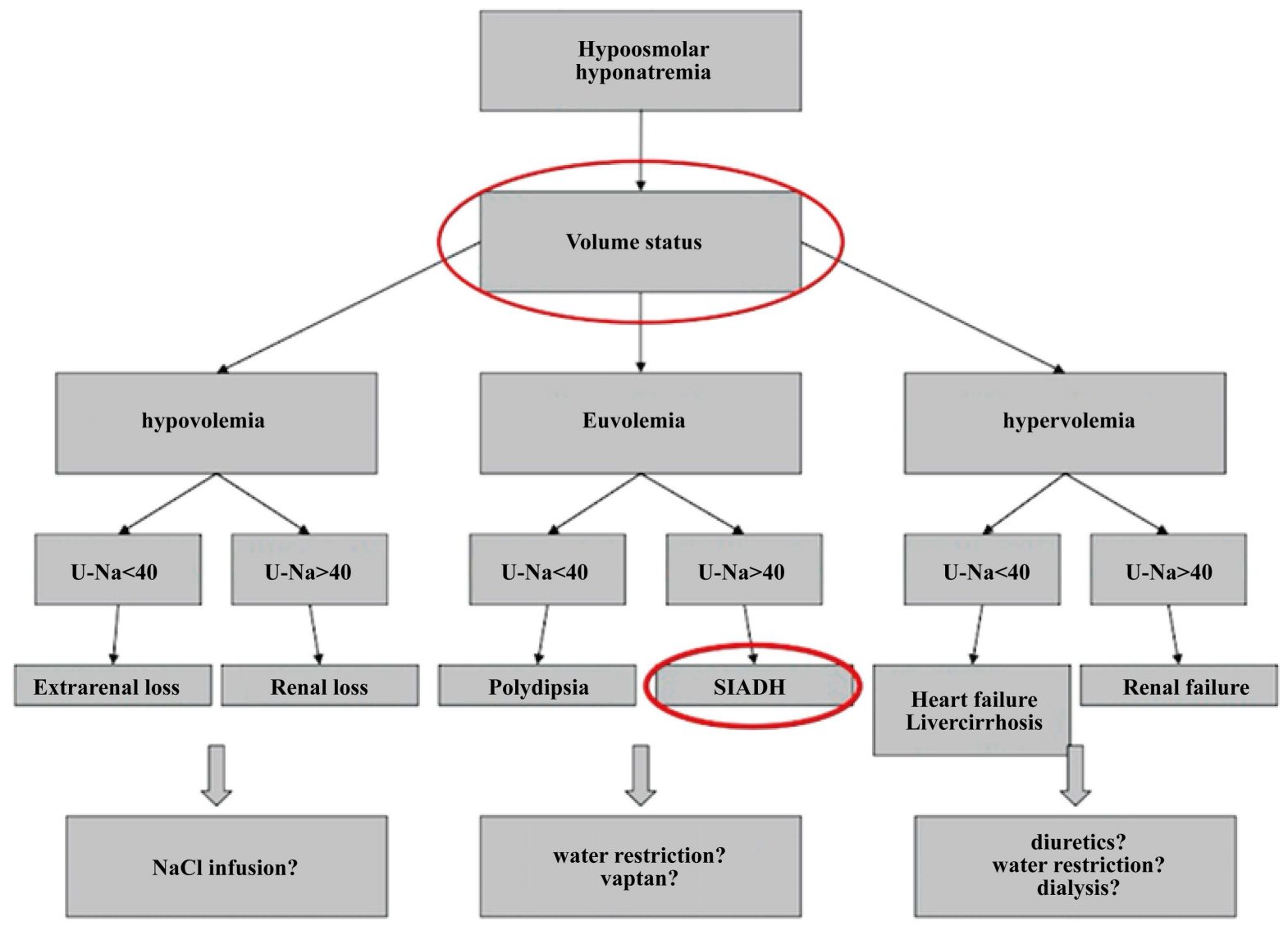

Figure 1. Diagnostic algorithm for hyponatremia. 
(proET-1) and N-terminal pro-brain natriuretic peptide (NT-proBNP) are measured in plasma samples drawn at the entry to the Emergency Department.

\section{Methods}

\subsection{Subjects}

Blood samples were collected from an unselected patient population at entry to the Emergency Departments in Lund and Malmö, Sweden, regardless of reason for the hospital visit and medical specialty (internal medicine, surgery, orthopedics, neurology or trauma). If plasma-sodium (P-Na) was $\leq 125 \mathrm{mmol} / \mathrm{L}$ the sample was frozen for further analysis of biomarkers. The plasma sample was thus obtained before any treatment was initiated.

Only patients who were admitted for in-hospital care were included in the study to ensure a solid foundation for subsequent evaluation. Inclusions were made between Sept. 2009 and May 2010. If hyperglycemia was present a corrected sodium level was calculated and the patient was only included if this value was $\leq 125$ $\mathrm{mmol} / \mathrm{L}[5]$.

Patients were included after they had signed a written consent. The study was also approved by the Ethics Committee in Lund.

\subsection{Record Review and Classification}

Patient records were examined after discharge from the hospital. A thorough assessment of volume status, treatment effect and etiology was made using all available information in the patient records including plasmaand urine-analyses. As no criteria based gold standard exist for assessment of volume status, the categorization of volume status was based on the judgment by the specialist who reviewed all available information in the patient record and evaluation was performed blinded for biomarker results. In cases where information on all criteria of SIADH was missing the diagnosis was made judiciously by the authors.

\subsection{Laboratory Measurements}

The plasma samples taken from patients at entry to the Emergency Department were directly frozen and stored in $-80^{\circ} \mathrm{C}$. Biomarkers of cardiovascular load (MR-proANP, NT-proBNP, pro Endothelin-1 and MR-ADM) and osmotic stress (copeptin) of interest in the pathogenesis of hyponatremia were examined. Mid-regional pro-atrial natriuretic peptide (MR-proANP), copeptin, mid-regional pro-adrenomedullin (MR-proADM) and pro-endothelin-1 (proET-1) were measured on a fully automated immunoassay system (Kryptor, Thermo Fisher) using methods described previously [6]-[9]. N-terminal pro-brain natriuretic peptide (NT-pro BNP) was determined using the Dimension RxL N-BNP (Dade-Behring, Germany) [10].

Median MR-proANP of 325 healthy individuals in previous investigations was $45 \mathrm{pmol} / \mathrm{L}$ (interassay CV at $45 \mathrm{pmol} / \mathrm{L}=10 \%$ ), and the 97.5 th percentile (upper limit of reference interval) was $163.9 \mathrm{pmol} / \mathrm{L}$ (interassay $\mathrm{CV}$ at $163.9 \mathrm{pmol} / \mathrm{L}=7.5 \%$ ) [9].

Median CT-proAVP in 359 healthy individuals in previous investigations was $4.2 \mathrm{pmol} / \mathrm{L}$ and the range between the 2.5th and 97.5th percentile was $1.7-11.3 \mathrm{pmol} / \mathrm{L}$ (Interassay CV at $4.2 \mathrm{pmol} / \mathrm{L}=14 \%$, interassay CV at $11.25 \mathrm{pmol} / \mathrm{L}=9 \%$ ) [6].

The normal range for MR-pro-ADM is $0.17-0.49 \mathrm{nmol} / \mathrm{L}$ (interassay CV was $<20 \%$ for values $>0.12 \mathrm{nmol} / \mathrm{L}$ ) and for CT pro-ET1 24.8 - $66.6 \mathrm{pmol} / \mathrm{L}$ (interassay CV $<10 \%$ for values $>10 \mathrm{pmol} / \mathrm{L}$ ) [11] [12]. The reference range for the NT-proBNP assay is $<300 \mathrm{ng} / \mathrm{L}$ in patients $>60$ years and the interassay CV $4.6 \%$ [13].

\subsection{Statistics}

Data was analysed with the SPSS statistical software (version 19, SPSS Inc., Chicago, Ill.)

Groupwise differences in continuous and categorical descriptive variables were calculated with Mann-Whitney U-test and Chi Square Test or Fisher's exact test, when appropriate. For the analysis of biomarkers we used crude and multivariate linear regression models with backward elimination of covariates ( $p$-value for retention $<0.10$ ) with groups of gradually increasing volemic status (hypovolemia $=0$, euvolemia $=1$ and hypervolemia $=$ 2 ) as the dependent variable. In the comparison between two groups of volemic status and when SIADH patients were compared with other causes of hyponatremia we used crude and multivariate logistic regression with back- 
ward elimination of covariates ( $p$-value for retention $<0.10$ ).

Due to skewed distributions, all biomarkers were ln-transformed before being entered into the linear and logistic regressions.

\section{Results}

\subsection{Patient Characteristics}

A total of 81 patients were included in the study between Sept 2009 and May 2010.

Hypertension, diabetes mellitus, heart failure and a history of previous $\mathrm{P}-\mathrm{Na} \leq 125 \mathrm{mmol} / \mathrm{L}$ were commonly occurring conditions. Ongoing treatment with diuretics, angiotensin-receptor blockers, ACE-inhibitors and antidepressants was also frequently noted (Table 1).

A well substantiated volemic state (hypo-, eu- or hypovolemia) could be established in 72 of the 81 patients. For that reason all analysis involving volume groups were performed in this subgroup. Due to insufficient plasma volumes, NT-proBNP could only be analysed in 64 of these patients. Analysis regarding NT-proBNP and volemic status was consequently executed in this smaller sample. The entire patient population was used in all other analyses. There was no significant difference in patient characteristics between those with and without data on volemic status or among those with and without data on volemic status and NT-proBNP (data not shown).

\subsection{Etiologies}

In patients in whom volemic state could be established $(n=72)$, hypovolemia was noted in $29 \%$, euvolemia in $53 \%$ and hypervolemia in $18 \%$ of the patients (Table 2).

The underlying cause of hyponatremia was often found to be multifactorial. Up to three etiologies were recognized in some patients but most patients had one or two underlying causes (one etiology, n (\%): 31 (43), two etiologies: 32 (44), three etiologies: 8 (1)). The most common underlying causes of hyponatremia were SIADH (33\%), sodium and water depletion due to gastrointestinal losses, diuretics, malnutrition or Addison's disease $(60 \%)$ and sodium and water expansion caused by heart failure, liver cirrhosis or kidney failure (15\%) (Table 2).

\subsection{Biomarkers of Volemic State and SIADH}

\subsubsection{MR-proANP}

In crude linear regression analysis a significant association was observed between ln-transformed values of MR-proANP levels and volemic state (beta-coefficient 0.30 per SD increment of ln-transformed MR-proANP per volemia class, 95\% CI $0.15-0.44, p=0.0001$ ) (Table 3, Figure 2(a)). In a multivariate backward elimination model (entering age, gender, thiazide or loop diuretics, heart failure, cirrhosis, oedema, gastrointestinal losses and MR-proANP). MR-proANP remained significantly related to volemic state (beta-coefficient 0.18 per SD increment of MR-proANP per volemia class, 95\% CI $0.04-0.32, p=0.012$ ).

As the distinction between mild hypovolemia and euvolemia is the clinically more important issue, data was also analysed with respect to hypo- or euvolemia $(n=59)$ using logistic regression. In a crude analysis, MRproANP was significantly related to euvolemia (OR: 2.54 per SD increment of MR-proANP, 95\% CI 1.32 - 4.86, $p=0.005$ ) and remained so after the multivariate backward elimination modelling (OR: 2.45 per SD of MR-proANP, 95\% CI 1.22 - 4.91, $p=0.012$ ) (Table 3).

\subsubsection{NT-proBNP}

NT-proBNP is the most extensively studied natriuretic peptide and is already in widespread use in clinical practice, primarily for screening and treatment monitoring of heart failure. The association between NT-proBNP levels and volemic states was thus examined. Ln-transformed values of NT-proBNP were significantly associated with volemia class in crude analysis (beta-coefficient 0.23 per SD increment of ln-transformed NT-proBNP per volemia class, 95\% CI $0.07-0.38, p=0.006$ ) but did not remain so after the multivariate backward elimination modelling. In contrast to MR-proANP, NT-proBNP had no significant relationship with the more clinically relevant comparison between hypo- and euvolemia (OR: 1.54 per SD of NT-proBNP, 95\% CI $0.84-2.82, p=$ 0.159) (Table 3, Figure 2(b)). 
Table 1. Patient characteristics at admission.

\section{Median (IQR) or $\%(n) n=81$}

\begin{tabular}{|c|c|}
\hline Age (yrs) & $76(66-87)$ \\
\hline Gender ( $\%$ females) & $65(53)$ \\
\hline P-Na at admission $(\mathrm{mmol} / \mathrm{L})$ & $119(114-122)$ \\
\hline Systolic blood pressure & $140(130$ - 169) \\
\hline Diastolic blood pressure & $76(65-90)$ \\
\hline \multicolumn{2}{|l|}{ Morbidities: } \\
\hline Previous $\mathrm{P}-\mathrm{Na}<125 \mathrm{mmol} / \mathrm{L}$ & $30(24)$ \\
\hline Alcohol abuse & $12(10)$ \\
\hline Hypertension & $63(51)$ \\
\hline Heart failure & $17(14)$ \\
\hline Kidney failure & $5(4)$ \\
\hline Diabetes mellitus & $19(15)$ \\
\hline Liver cirrhosis & $4(3)$ \\
\hline Malignancy & $11(9)$ \\
\hline \multicolumn{2}{|l|}{ Drug treatment: } \\
\hline Thiazide diuretics & $32(26)$ \\
\hline Loop diuretics & $24(19)$ \\
\hline Spironolactone & $14(11)$ \\
\hline Potassium sparing diuretics & $10(8)$ \\
\hline ARB & $20(16)$ \\
\hline ACEi & $11(9)$ \\
\hline SSRI & $14(11)$ \\
\hline Tricyclic antidepressants & $1(1)$ \\
\hline Mirtazapine & $6(5)$ \\
\hline Opioids & $6(5)$ \\
\hline Antiepileptics & $5(4)$ \\
\hline Cortisone & $9(7)$ \\
\hline S-osm (mOsm/kg, n = 31) & $262(250-272)$ \\
\hline U-osm (mOsm/kg, n = 37) & $350(262-504)$ \\
\hline U-Na (mmol/L, n = 32) & $60(28-80)$ \\
\hline S-cortisol (nmol/L, n = 45) & $543(438-857)$ \\
\hline $\mathrm{TSH}(\mathrm{mU} / \mathrm{L}, \mathrm{n}=63)$ & $2(1-10)$ \\
\hline Krea $(\mu \mathrm{mol} / \mathrm{L}, \mathrm{n}=80)$ & $71(55-90)$ \\
\hline $\mathrm{CRP}(\mathrm{mg} / \mathrm{L}, \mathrm{n}=80)$ & $14(2-45)$ \\
\hline Glucose $(\mathrm{mmol} / \mathrm{L}, \mathrm{n}=76)$ & $7(6-9)$ \\
\hline
\end{tabular}

ACEi = angiotensin converting enzyme inhibitors; ARB = angiotensin receptor blockers; SSRI = selective serotonin reuptake inhibitors. 
Table 2. Underlying causes.

\section{All patients $(n=81)$}

\begin{tabular}{|c|c|c|c|}
\hline & \multicolumn{3}{|c|}{$\%$} \\
\hline Sodium and water depletion & \multicolumn{3}{|c|}{54} \\
\hline SIADH & \multicolumn{3}{|c|}{30} \\
\hline Sodium and water expansion & \multicolumn{3}{|c|}{14} \\
\hline Polydipsia & \multicolumn{3}{|c|}{11} \\
\hline Alcohol abuse & \multicolumn{3}{|c|}{11} \\
\hline \multirow[t]{4}{*}{ Hyperglycemia } & \\
\hline & \multicolumn{3}{|c|}{ Patients with defined volume status $(n=72)$} \\
\hline & \multicolumn{3}{|c|}{$\%^{* *}(\mathbf{n})^{*}$} \\
\hline & Hypovolemia (21) & Euvolemia (38) & Hypervolemia (13) \\
\hline Sodium and water depletion & $90(19)$ & $58(22)$ & $15(2)$ \\
\hline SIADH & $14(3)$ & $47(18)$ & $23(3)$ \\
\hline Sodium and water expansion & 0 & $5(2)$ & $69(9)$ \\
\hline Polydipsia & $10(2)$ & $11(4)$ & $23(3)$ \\
\hline Alcohol abuse & $24(5)$ & $5(2)$ & $15(2)$ \\
\hline Hyperglycemia & $5(1)$ & $11(4)$ & $8(1)$ \\
\hline
\end{tabular}

${ }^{*}$ More than one diagnosis possible; ${ }^{* *} \%$ of patients in the volume status group with the specific etiological diagnosis.

Table 3. Biomarkers and subdivision of hyponatremia.

\begin{tabular}{cccc}
\hline & \multicolumn{2}{c}{ Volume status } & \multicolumn{2}{c}{ SIADH vs other causes } \\
\hline & Hypo/eu/hypervolemi (p-value) & Hypo vs euvolemia (p-value) $^{* * *}$ & $\left(^{(p-v a l u e)^{* * *}}\right.$ \\
\hline ET-1 & 0.352 & 0.772 & 0.457 \\
MR-proADM & 0.625 & 0.337 & 0.479 \\
MR-proANP & $\boldsymbol{p}<\mathbf{0 . 0 0 1}$ & $\mathbf{0 . 0 0 5}$ & 0.971 \\
NT-proBNP & $\boldsymbol{p}<\mathbf{0 . 0 0 6}$ & 0.159 & 0.282 \\
Copeptin & 0.875 & 0.371 & 0.286 \\
\hline
\end{tabular}

${ }^{*}$ Crude linear regression; ${ }^{* *}$ Crude logistic regression.

\subsubsection{Copeptin}

The median copeptin level in the entire study population was 24.7 pmol/L (IQR 8.5 - 75.4) compared to 31.99 $\mathrm{pmol} / \mathrm{L}$ (IQR 6.6 - 96.8) in patients with SIADH (NS) (Table 4).

No linear association was found between copeptin values and volemic state (Table 3) (Figure 3).

\subsection{Other Biomarkers}

Mid-regional pro-adrenomedullin (MR-proADM) andproendothelin-1 (proET-1) was also analysed. No association was found between biomarker levels and volemic state. There was no significant difference between any of the other biomarkers and the presence or absence of SIADH (Table 3). 


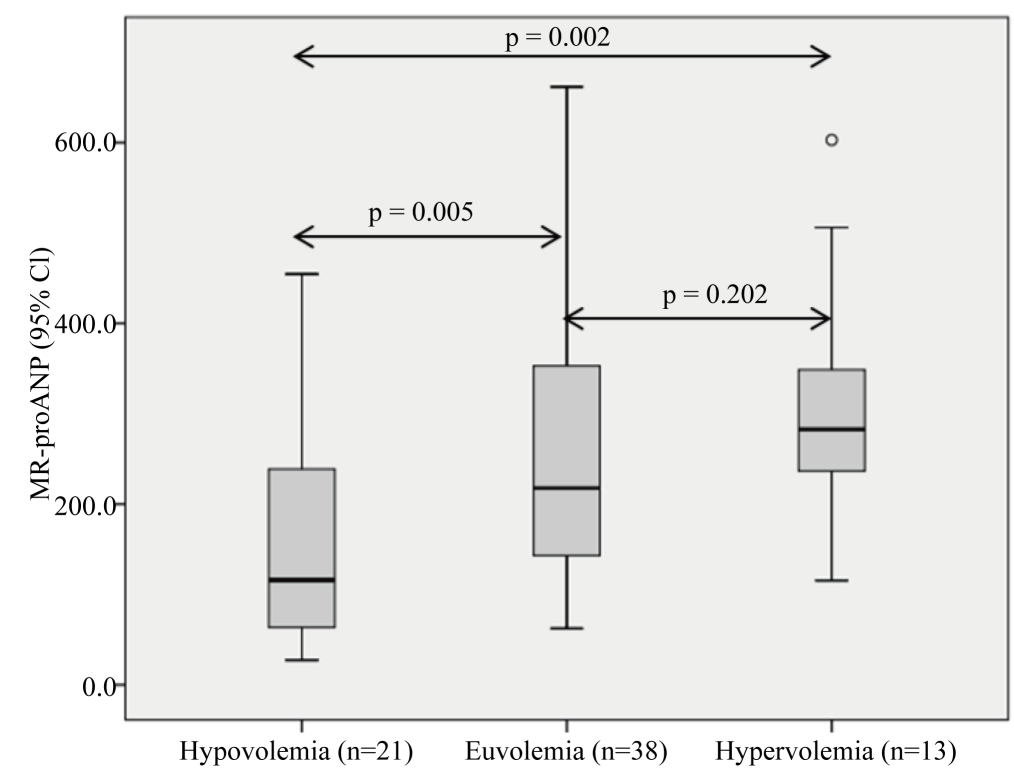

(a)

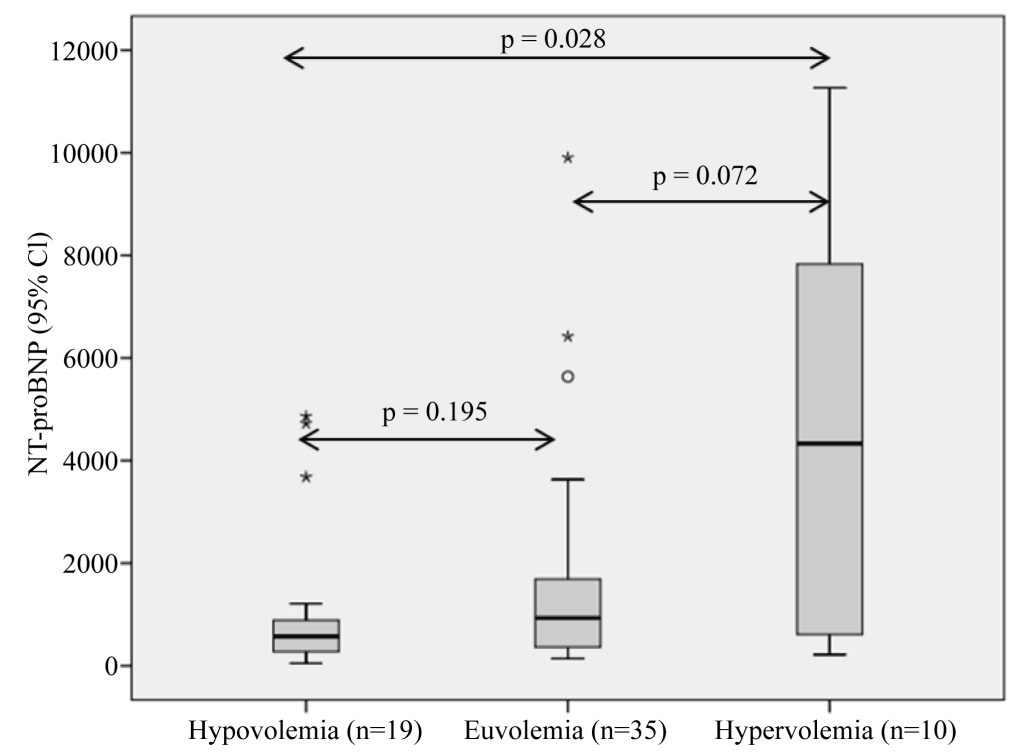

(b)

Figure 2. MR-proANP and NT-proBNP levels in different volemic states boxplot with median values, interquartile ranges and outliers.

Table 4. Biomarker levels.

\begin{tabular}{cc}
\hline & Median (IQR) \\
\hline MR-proANP $(\mathrm{pmol} / \mathrm{L})$ & $215(116-339)$ \\
MR-proADM $(\mathrm{nmol} / \mathrm{L})$ & $0.90(0.58-1.28)$ \\
$\operatorname{proET}-1(\mathrm{pmol} / \mathrm{L})$ & $55(40-72)$ \\
Copeptin $(\mathrm{pmol} / \mathrm{L})$ & $24.7(8.5-75.4)$ \\
NT-proBNP $(\mathrm{ng} / \mathrm{L}, \mathrm{n}=72)$ & $837(359-2631)$ \\
\hline
\end{tabular}

$n=81$ if not otherwise specified. 


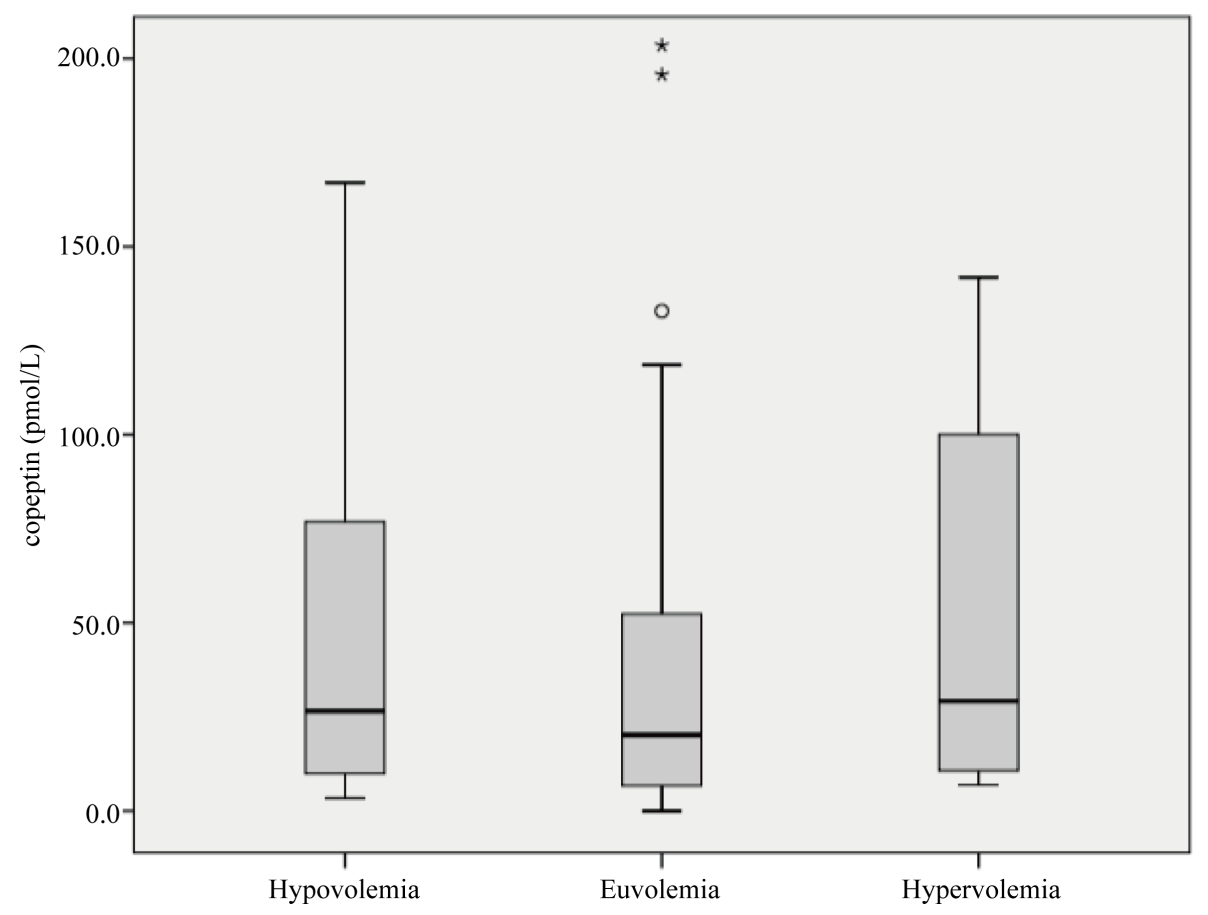

Figure 3. Copeptin levels in different volemic states.

\section{Discussion}

A swift and accurate evaluation of volume status is crucial to the early management of hyponatremia. The distinction between hypovolemia, euvolemia and hypervolemia determines the treatment strategy. Several previous studies have described how cumbersome and inaccurate clinical assessments of volume status can be, with low sensitivity and specificity [3] [4]. The urine analysis of osmolality and sodium add valuable information but takes time to collect. The widespread use of diuretics in this elderly patient group risks influencing results of urine analysis, making them inaccurate. Previous studies have observed a low frequency of laboratory evaluations [14]-[16]. In a Swedish study only 31\% of patients with P-Na $\leq 120 \mathrm{mmol} / \mathrm{L}$ underwent analysis of plasma osmolality and urine analysis of sodium and osmolality [1]. New diagnostic tools are therefore needed.

Hyponatremia is often the result of multiple coexisting factors [16] [17]. Several cardiovascular, inflammatory and volume regulating systems are involved. In this study we examined the diagnostic value of specific biomarkers in: 1) the assessment of volume status and 2) the diagnosis of SIADH.

\subsection{Volume Status}

The results indicate that MR-proANP may be a promising biomarker for assessment of volume status as MRproANP levels were significantly associated with volemic state (hypovolemia, euvolemia or hypervolemia). Even more importantly the level of MR-proANP could distinguish hypovolemia from euvolemia. This categorization poses the greatest challenge in clinical practice and affects treatment. MR-proANP was significantly related to euvolemia with an odds ratio of 2.5 per SD of MR-proANP compared to hypovolemia and the correlation remained significant after multivariate analysis.

Since NT-proBNP is already in widespread use in clinical practice the same analysis was performed using NT-proBNP levels. Interestingly enough, no significant correlation was found between NT-proBNP and euvolemia versus hypovolemia.

The concentration of ANP in the circulation is 10- to 50-fold higher than the BNP concentration, which could explain why MR-proANP was superior to NT-proBNP in the difficult distinction between euvolemia and hypovolemia. Comparisons between MR-proANP and NT-proBNP in the diagnosis of heart failure have shown a high diagnostic accuracy of MR-proANP. It has even been postulated that MR-proANP may provide additional diagnostic information in patients with grey zone NT-proBNP levels [18]. 
Even so MR-proANP has not been thoroughly investigated in conjunction with volemic states in hyponatremia. In a small study of 40 patients by Coenraad et al., significantly lower ANP concentrations were found in hypovolemic and euvolemic subjects with hyponatremia compared to controls, whereas hypervolemia was associated with higher levels. No significant difference was noticed in this study between hypovolemic and euvolemic patients [19]. More data exist in cardiovascular studies on orthostatic stress where a reduction in ANP levels has been observed in head-up tilt tests [20] [21]. These studies indicate a possible effect on MR-proANP levels even in mild hypovolemia. Findings which could be of interest in conjunction to the examination of hyponatremic patients.

Vasopressin is the key hormone in the regulation of osmolality but the hormone itself is unfortunately difficult to measure due to in vitro instability. Copeptin is the C-terminal fragment of the vasopressin prohormone and it is secreted in equimolar amounts from the pituitary. This glycosylated peptide is stable and can be analysed easier [22]. Hence it is a perfect surrogate marker for vasopressin levels.

Copeptin levels were not found to be significantly associated with volemic status. In spite of that there was a tendency of higher copeptin levels in hypo- and hypervolemia compared to euvolemia. This may indicate a non-osmotic stimulation of vasopressin through baroreceptors.

\subsection{Diagnosis of SIADH}

Vasopressin is of special interest in patients with SIADH, where it's effect on osmolarity andvolume status is believed to be pivotal. Non-osmotic stimulation of vasopressin secretion through baroreceptors is exaggerated in patients with SIADH thereby overriding the effect of decreased osmolarity. Also, in our patients at the Emergency Department stimulation on vasopressin release is increased in the majority of acutely ill patients. Copeptin levels were indeed elevated in the entire study population overshadowing raised levels in patients as clinically judged with SIADH (median $25 \mathrm{pmol} / \mathrm{l}$ vs $32 \mathrm{pmol} / \mathrm{l}$, NS) (Table 4). This increase in acute ill patients is in consert with previous studies, where Copeptin in critically ill patients increased to several hundred pmol/l as compared to healthy volunteers showing a mediumlevel of 4 pmol/l [6] [23]-[27].

Our study was performed in a full clinical setting, where the patient was considered as belonging to a specific volume status group (hypo-, eu- and hypervolemia) based on laboratory results at admission, symptoms and signs, underlying conditions and evaluation of treatment response. Lacking a gold standard for volume status, in the absence of invasive measurements only performed at the Intensive Care Unit, our classification was accordingly based on the available clinical and laboratory information in routine care management at our Emergency Department at this time period.

For the diagnosis of SIADH we used the criteria originally described by Bartter et al. [28]. However, in cases where complete information was lacking, the diagnosis of SIADH was made judiciously after discussion among the investigators blinded for biomarker results.

Two previous studies have investigated copeptin in the differential diagnosis of hyponatremia.

In the article by Fenske et al. from 2009 copeptin levels could identify patients with primary polydipsia but had limited value in the differential diagnosis of other hyponatremic disorders [7]. Nigro et al. also examined copeptin levels in hyponatremic patients admitted to hospital with lower respiratory tract infections or acute cerebrovascular events [29].

In patients with respiratory infections the median copeptin level was $32 \mathrm{pmol} / \mathrm{L}$ (IQR 22-50). Uncorrected, there was a significant difference in copeptin values across different volume status groups with increasing levels with increasing extracellular fluid volumes. After correction for multiple testing however the difference between hypovolemia and euvolemia did not remain significant. In the group with acute cerebrovascular events no significant association was found between neither copeptin and volume status nor copeptin and etiology.

Our study further indicates that copeptin add little information in the initial differential diagnosis of hyponatremic disorders.

Our study has limitations. The study was observational and therefore firm conclusions regarding causality between suspected underlying causes cannot be drawn. The diagnosis of volume status and underlying causes of hyponatremia were based on hospital records and laboratory databases with the inherent limitations. For example only 32 patients had urine analysis of sodium performed and the diagnosis had to be made judiciously on available data. The lack of an objective diagnosis of volume status should be taken into account when interpreting the data. 
We emphasize that alcohol abuse as such is not a recognized single etiology however we chose to include it in accordance with previous studies as hyponatremia is common in patients with alcohol abuse through several different mechanisms [15] [30] [31].

Introduction of biomarkers such as MR-proANP should be preceeded by studies testing if it is superior in a setting where urine sodium is always analyzed. In the current study we could not assess this as U-Na was missing in many patients. Furthermore it should be stressed that there was a large overlap of MR-proANP levels between volume status groups. Thus other biomarkers than MR-proANP will be necessary to identify in order to provide added value in the clinic. This is a small hypothesis generating study and since there is no gold standard for evaluation of volume status we prefer only presenting our findings of statistically significant relations, i.e. omitting possible cut-off limits for MR-proANP.

\section{Conclusion}

MR-proANP is a promising biomarker for distinguishing between hypovolemia and euvolemia in hyponatremic patients. Copeptin levels alone cannot diagnose SIADH in the Emergency Department. A more intricate diagnostic algorithm would have to be used where an evaluation of the vasopressin system could be part of a wider laboratory and clinical evaluation. The limitations of the study should be taken into account when considering the results but the findings suggest that further confirmatory studies are needed.

\section{Declaration of Interests}

Dr. Olsson and Dr. Öhlin have served as consultants in educational activities arranged by Otsuka Pharma Scandinavia AB.

\section{Funding}

The study is supported by the European Research Council (StG-282255), the Swedish Heart and Lung Foundation, Swedish Research Council; the Novo Nordisk Foundation, the Skåne University Hospital donation funds; the Medical Faculty, Lund University; the Governmental funding of clinical research within the national health services, the Albert Påhlsson Research Foundation, Region Skåne, the King Gustav V and Queen Victoria Foundation and the Marianne and Marcus Wallenberg Foundation.

\section{Acknowledgements}

We would like to thank Kerstin Roos, Peter Almgren and Philippe Burri for their invaluable help.

\section{References}

[1] Olsson, K., Öhlin, B. and Melander, O. (2013) Epidemiology and Characteristics of Hyponatremia in the Emergency Department. European Journal of Internal Medicine, 24, 110-116. http://dx.doi.org/10.1016/j.ejim.2012.10.014

[2] Upadhyay, A., Jaber, B.L. and Madias, N.E. (2006) Incidence and Prevalence of Hyponatremia. American Journal of Medicine, 119, 30-35. http://dx.doi.org/10.1016/j.amjmed.2006.05.005

[3] Chung, H.M., Kluge, R., Schrier, R.W. and Anderson, R.J. (1987) Clinical Assessment of Extracellular Fluid Volume in Hyponatremia. American Journal of Medicine, 83, 905-908. http://dx.doi.org/10.1016/0002-9343(87)90649-8

[4] Musch, W., Thimpont, J., Vandervelde, D., Verhaeverbeke, I., Berghmans, T. and Decaux, G. (1995) Combined Fractional Excretion of Sodium and Urea Better Predicts Response to Saline in Hyponatremia than Do Clinical and Biochemical Parameters. American Journal of Medicine, 99, 348-355. http://dx.doi.org/10.1016/S0002-9343(99)80180-6

[5] Hillier, T.A., Abbott, R.D. and Barrett, E.J. (1999) Hyponatremia: Evaluating the Correction Factor for Hyperglycemia. American Journal of Medicine, 4, 399-403. http://dx.doi.org/10.1016/S0002-9343(99)00055-8

[6] Morgenthaler, N.G., Struck, J., Alonso, C. and Bergmann, A. (2006) Assay for the Measurement of Copeptin, a Stable Peptide Derived from the Precursor of Vasopressin. Clinical Chemistry, 52, 112-119. http://dx.doi.org/10.1373/clinchem.2005.060038

[7] Fenske, W., Stork, S., Blechschmidt, A., Maier, S.G., Morgenthaler, N.G. and Allolio, B. (2009) Copeptin in the Differential Diagnosis of Hyponatremia. Journal of Clinical Endocrinology and Metabolism, 94, 123-129. http://dx.doi.org/10.1210/jc.2008-1426 
[8] Caruhel, P., Mazier, C., Kunde, J., Morgenthaler, N.G. and Darbouret, B. (2009) Homogeneous Time-Resolved Fluoroimmunoassay for the Measurement of Midregional Proadrenomedullin in Plasma on the Fully Automated System B.R.A.H.M.S KRYPTOR®. Clinical Biochemistry, 42, 725-728. http://dx.doi.org/10.1016/j.clinbiochem.2009.01.002

[9] Morgenthaler, N.G., Struck, J., Thomas, B. and Bergmann, A. (2004) Immunoluminometric Assay for the Midregion of Pro-Atrial Natriuretic Peptide in Human Plasma. Clinical Chemistry, 50, 234-236. http://dx.doi.org/10.1373/clinchem.2003.021204

[10] Di Serio, F., Ruggieri, V., Varraso, L., De Sario, R., Mastrorilli, A. and Pansini, N. (2005) Analytical Evaluation of the Dade Behring Dimension RxL Automated N-Terminal proBNP (NT-proBNP) Method and Comparison with the Roche Elecsys 2010. Clinical Chemistry and Laboratory Medicine, 43, 1263-1273. http://dx.doi.org/10.1515/CCLM.2005.217

[11] Morgenthaler, N.G., Struck, J., Alonso, C. and Bergmann, A. (2005) Measurement of Midregional Proadrenomedullin in Plasma with an Immunoluminometric Assay. Clinical Chemistry, 51, 1823-1829. http://dx.doi.org/10.1373/clinchem.2005.051110

[12] Papassotiriou, J., Morgenthaler, N.G., Struck, J., Alonso, C. and Bergmann, A. (2006) Immunoluminometric Assay for Measurement of the C-Terminal Endothelin-1 Precursor Fragment in Human Plasma. Clinical Chemistry, 52, 1144-1151. http://dx.doi.org/10.1373/clinchem.2005.065581

[13] Januzzi, J., Kimmenade, R., Lainchbury, J., Bayes-Genis, A., Ordonez-Lianos, J. and Santalo-Bel, M. (2006) NTproBNP Testing for Diagnosis and Short-Term Prognosis in Acute Destabilized Heart Failure: An International Pooled Analysis of 1256 Patients. European Heart Journal, 27, 330-337. http://dx.doi.org/10.1093/eurheartj/ehi631

[14] Saeed, B.O., Beaumont, D., Handley, G.H. and Weaver, J.U. (2002) Severe Hyponatremia: Investigation and Management in a District General Hospital. Journal of Clinical Pathology, 55, 893-896. http://dx.doi.org/10.1136/jcp.55.12.893

[15] Huda, M.S., Boyd, A., Skagen, K., Wile, D., van Heyningen, C., Watson, I., Wong, S. and Gill, G. (2006) Investigation and Management of Severe Hyponatremia in a Hospital Setting. Postgraduate Medical Journal, 82, 216-219. http://dx.doi.org/10.1136/pmj.2005.036947

[16] Clayton, J.A., Le Jeune, I.R. and Hall, I.P. (2006) Severe Hyponatremia in Medical In-Patients: Aetiology, Assessment and Outcome. Quarterly Journal of Medicine, 99, 505-511. http://dx.doi.org/10.1093/qjmed/hcl071

[17] Shapiro, D.S., Sonnenblick, M., Galperin, I., Melkonyan, L. and Munter, G. (2010) Severe Hyponatraemia in Elderly Hospitalized Patients: Prevalence, Aetiology and Outcome. Internal Medicine Journal, 40, 574-580. http://dx.doi.org/10.1111/j.1445-5994.2010.02217.x

[18] Potocki, M., Breidthardt, T., Reichlin, T., Hartwiger, S., Morgenthaler, N.G., Bergmann, A., Noveanu, M., Freidank, H., Taegtmeyer, A.B., Wetzel, K., Boldanova, T., Stelzig, C., Bingisser, R., Christ, M. and Mueller, C. (2010) Comparison of Midregional Pro-Atrial Natriuretic Peptide with N-Terminal Pro-B-Type Natriuretic Peptide in the Diagnosis of Heart Failure. Journal of Internal Medicine, 267, 119-129. http://dx.doi.org/10.1111/j.1365-2796.2009.02135.x

[19] Coenraad, M.J., Bolk, J.H., Frölich, M. and Meinders, A.E. (2007) Plasma Arginine Vasopressin and Atrial Natriuretic Peptide Concentration in Patients with Hyponatremia at Diagnosis and Following Treatment. European Journal of Internal Medicine, 18, 221-229. http://dx.doi.org/10.1016/j.ejim.2006.11.008

[20] Roessler, A., Goswami, N., Haditsch, B., Loeppky, J.A., Lufts, F.C. and Hinghofer-Szalkay, H. (2011) Volume Regulating Hormone Responses to Repeated Head-Up Tilt and Lower Body Negative Pressure. European Journal of Clinical Investigation, 41, 863-869. http://dx.doi.org/10.1111/j.1365-2362.2011.02476.x

[21] Freitas, J., Santos, R., Azevedo, E., Carvalho, M. and Rocha-Goncalces, F. (2005) Neurohormonal Behaviour during Prolonged Orthostatic Stress in Normotensive Subjects. Revista Portuguesa de Cardiologia, 24, 81-86.

[22] Balanescu, S., Kopp, P., Gaskill, M.B., Morgenthaler, N.G., Schindler, C. and Rutishauser, J. (2011) Correlation of Plasma Copeptin and Vasopressin Concentrations in Hypo-, Iso- and Hyperosmolar States. Journal of Clinical Endocrinology and Metabolism, 96, 1046-1052. http://dx.doi.org/10.1210/jc.2010-2499

[23] Darzy, K., Dixit, K.C., Shalet, S.M., Morgenthaler, N.G. and Brabant, G. (2010) Circadian Secretion Pattern of Copeptin, the C-Terminal Vasopressin Precursor Fragment. Clinical Chemistry, 56, 1190-1191. http://dx.doi.org/10.1373/clinchem.2009.141689

[24] Maeder, M.T., Staub, D., Brutsche, M.H., Arenja, N., Socrates, T., Reiter, M., Meissner, J., Morgenthaler, N.G., Bergmann, A., Struck, J. and Mueller, C. (2010) Copeptin Response to Clinical Maximal Exercise Tests. Clinical Chemistry, 56, 674-676. http://dx.doi.org/10.1373/clinchem.2009.136309

[25] Khan, S.Q., Dhillon, O.S., O’Brien, R.J., Struck, J., Quinn, P.A., Morgenthaler, N.G., Squire, I.B., Davies, J.E., Bergmann, A. and Ng, L. (2007) C-Terminal Provasopressin (Copeptin) as a Novel and Prognostic Marker in Acute Myocardial Infarction. Circulation, 115, 2103-2110. http://dx.doi.org/10.1161/CIRCULATIONAHA.106.685503

[26] Bhandari, S., Loke, I., Davies, J., Squire, I.B., Struck, J. and Ng, L. (2009) Gender and Renal Function Influence 
Plasma Levels of Copeptin in Healthy Individuals. Clinical Science, 116, 257-263. http://dx.doi.org/10.1042/CS20080140

[27] Struck, J., Morgenthaler, N.G. and Bergmann, A. (2005) Copeptin, a Stable Peptide Derived from the Vasopressin Precursor, Is Elevated in Serum of Sepsis Patients. Peptides, 26, 2500-2504. http://dx.doi.org/10.1016/j.peptides.2005.04.019

[28] Bartter, F.C. and Schwartz, W.B. (1967) The Syndrome of Inappropriate Secretion of Antidiuretic Hormone. American Journal of Medicine, 42, 790-806. http://dx.doi.org/10.1016/0002-9343(67)90096-4

[29] Nigro, N., Morgenthaler, N.G., Fluri, F., Schuetz, P., Neidert, S., Stolz, D., Bingisser, R., Tamm, M., Christ-Crain, M. and Katan, M. (2011) The Use of Copeptin, the Stable Peptide of the Vasopressin Precursor, in the Differential Diagnosis of Sodium Imbalance in Patients with Acute Diseases. Swiss Medical Weekly, 141, w13270. http://dx.doi.org/10.4414/smw.2011.13270

[30] Gill, G., Huda, B., Boyd, A., Skagen, K., Wile, D., Watson, I. and van Heyningen, C. (2006) Characteristics and Mortality of Severe Hyponatremia-A Hospital Based Study. Clinical Endocrinology, 65, 246-249. http://dx.doi.org/10.1111/j.1365-2265.2006.02583.x

[31] Liamis, G., Milionis, H., Rizos, E., Siamopoulos, K. and Elisaf, M. (2000) Mechanism of Hyponatremia in Alcohol Patients. Alcohol \& Alcoholism, 35, 612-616. http://dx.doi.org/10.1093/alcalc/35.6.612

\author{
Abbreviations \\ ADH: antidiuretic hormone, vasopressin \\ MR-proANP: mid-regional pro-atrial natriuretic peptide \\ MR-proADM: mid-regional pro-adrenomedullin \\ proET-1: pro-endothelin-1 \\ NT-proBNP: N-terminal pro-brain natriuretic peptide
}

